ISSN 0103-9954

\title{
MECANISMOS DE REGENERAÇÃO NATURAL EM REMANESCENTE DE FLORESTA ESTACIONAL DECIDUAL
}

\author{
NATURAL REGENERATION MECHANISMS IN A SEASONAL DECIDUOUS \\ FOREST FRAGMENT
}

\author{
Marta Silvana Volpato Sccoti ${ }^{1}$ Maristela Machado Araujo ${ }^{2}$ \\ Cristiane Friedrich Wendler ${ }^{3}$ Solon Jonas Longhi ${ }^{2}$
}

\begin{abstract}
RESUMO
Diante da perda da biodiversidade e do habitat natural decorrentes da fragmentação das florestas, surge a necessidade de alternativas que possibilitem a recuperação desses ambientes. Assim, este estudo objetivou obter informações sobre o potencial dos mecanismos de regeneração natural (chuva de sementes, banco de sementes do solo, banco de plântulas e regeneração natural estabelecida) em um remanescente de Floresta Estacional Decidual, visando à conservação e recuperação destes ecossistemas. O estudo foi realizado de forma sistemática, a partir da demarcação de 14 unidades amostrais de $2.000 \mathrm{~m}^{2}$, dentro das quais foram selecionadas, aleatoriamente, 70 subparcelas onde foram avaliados os mecanismos de regeneração. A chuva de sementes foi avaliada durante um ano, com base no material obtido mensalmente a partir de coletores. Para o estudo do banco de sementes foram tomadas amostras de solo, com uso de um gabarito de ferro com $25 \times 25 \times 5 \mathrm{~cm}$ de dimensões, monitoradas durante 7 meses mediante germinação das sementes. A regeneração natural foi avaliada em duas classes: banco de plântulas e regeneração natural estabelecida, onde foram identificados e medidos indivíduos com $\mathrm{h} \geq 30 \mathrm{~cm}$ e $\mathrm{DAP}<5 \mathrm{~cm}$. A chuva de sementes apresentou densidade média de 1350 sementes. $\mathrm{m}^{-2}$ na área, dentre as quais se observou 73 espécies, predominantemente, arbóreas. No banco de sementes do solo, foram observadas 108 espécies, sendo $74 \%$ herbáceas. No banco de plântulas foram encontradas 48 espécies, sendo estas esciófilas e heliófilas, já na regeneração estabelecida foram encontradas 37 espécies, em sua maioria esciófilas. Concluiu-se que as espécies com maior potencial para perpetuar na área estudada foram Gymnanthes concolor, Sorocea bonplandii, Eugenia rostrifolia, Trichilia claussenii, Trichilia elegans e Dasyphyllum spinescens, sendo as mesmas indicadas para enriquecimento. Já as espécies Myrocarpus frondosus, Cupania vernalis, Nectandra megapotamica e Syagrus rommanzoffian apresentam certa restrição, dependendo de tratamentos silviculturais na floresta para garantir sua perpetuação na área.
\end{abstract}

Palavras-chave: chuva de sementes; banco de sementes do solo; banco de plântulas; regeneração natural estabelecida.

\begin{abstract}
Due to the loss of biodiversity and natural habitat because of the forests fragmentation, it is the needed to find alternatives that allow the recovery of these environments. Thus, this study aimed to obtain information about the potential of natural regeneration mechanisms (seed rain, soil seed bank, seedling bank and established natural regeneration) in a seasonal deciduous forest fragment in Santa Maria - RS state, in order to conserve and to recover these ecosystems. The study was conducted in a systematic way, having as a point of departure the demarcation of 14 sampling plots of $2000 \mathrm{sq} \mathrm{m}$. From those plots, 70 subplots were randomly selected to evaluate the natural regeneration mechanisms. The seed rain was evaluated
\end{abstract}

1. Engenheira Florestal, Doutoranda do programa de Pós-graduação em Engenharia Florestal, Centro de Ciências Rurais, Universidade Federal de Santa Maria, Av. Roraima, 1000, CEP 97105-900, Santa Maria (RS). martasccoti@yahoo.com.br

2. Engenheiros Florestais, Dr., Professores Associados do Departamento de Ciências Florestais, Centro de Ciências Rurais, Universidade Federal de Santa Maria, Av. Roraima, 1000, CEP 97105-900, Santa Maria (RS).

3. Acadêmica do curso de Graduação em Engenharia Florestal, Centro de Ciências Rurais, Universidade Federal de Santa Maria, Av. Roraima, 1000, CEP 97105-900, Santa Maria (RS).

Recebido para publicação em 12/11/2009 e aceito em 25/08/2010 
during one year based on the material that was monthly collected and analyzed from the collectors. To the study of the soil seed bank, soil samplings of $25 \mathrm{~cm} \times 25 \mathrm{~cm} \times 5 \mathrm{~cm}$ were taken and the collected material was monitored during 7 months, observing the seed germination. The natural regeneration was evaluated in two classes: seedling bank and established natural regeneration, where individuals $\mathrm{h} \geq 30 \mathrm{~cm}$ and $\mathrm{DBH} \leq$ $5 \mathrm{~cm}$ were identified and measured. The seed rain presented medium density of 1350 seeds $\mathrm{m}^{-2}$, in which 73 species, predominantly arboreal, were observed. In the soil seed bank, 108 species were observed, in which $74 \%$ were herbaceous. In the seedling bank, 48 species were found and they were heliophilous and sciaphilous species, while in the established natural regeneration, 37 species were found, prevailing sciaphilous. This study concluded that the species with the greatest potential to perpetuate in the studied area were Gymnanthes concolor, Soroceae bonplandii, Eugenia rostrifolia, Trichilia claussenii, Trichilia elegans and Dasyphylum spinescens, and they are highly indicated to the enrichment of the area. The species Myrocarpus frondosus, Cupania vernalis, Nectandra megapotamica and Syagrus rommanzoffiana showed certain restriction, depending on the silvicultural treatments in the forest to assure their perpetuation in the area.

Keywords: seed rain; soil seed bank; seedling bank; established natural regeneration.

\section{INTRODUÇ̃̃O}

No estado do Rio Grande do Sul, as áreas de florestas passaram por um processo de fragmentação decorrente da atividade humana. Processo esse que gerou, ao longo do tempo, perda da diversidade biológica e do habitat natural da fauna e da flora.

As alternativas de recuperação dessas áreas ocorrem, muitas vezes, a partir de projetos de reflorestamentos, porém, a floresta possui mecanismos próprios de recuperação e manutenção de sua diversidade, como por exemplo, os mecanismos de regeneração natural, que compreendem a chuva de sementes, o banco de sementes do solo e o banco de plântulas.

A chuva de sementes relaciona-se à dispersão de diásporos e a área abrangida por esse processo, que subsidia o estabelecimento da plântula (ALMEIDA-CORTEZ, 2004).

De acordo com Martinez-Ramos e SotoCastro (1993), a chuva de sementes é a maior fonte de propágulos para a regeneração. A disseminação de sementes que ocorre na floresta é composta por sementes produzidas no local e em áreas vizinhas. As sementes que são provenientes do próprio local promovem a autorregeneração da floresta, e aquelas trazidas por agentes dispersores representam o aumento da diversidade florística e genética.

O banco de sementes do solo é um conjunto de sementes viáveis encontradas na superfície e enterradas no solo (GARWOOD, 1989; ALMEIDACORTEZ, 2004), que são potencialmente capazes de substituir plantas adultas que morrem (BAKER, 1989).

Conforme Garwood (1989), o período de tempo em que as sementes permanecem no solo é determinado por fatores fisiológicos (germinação, dormência e viabilidade) e ambientais (umidade, temperatura, luminosidade, presença de predadores de sementes e patógenos). A autora ainda classifica o banco de sementes como transiente, podendo este ser de dois tipos: com sementes de vida curta, que não apresentam dormência e germinam, geralmente, no mesmo ano em que foram dispersadas; ou persistentes, representado por sementes dormentes que permanecem viáveis no solo por mais de um ano. Segundo Simpson et al. (1989), as sementes persistentes promovem uma reserva do potencial genético acumulado, tendo importante função na manutenção da diversidade genética das comunidades e populações.

O início da fase de plântula pode ser definido pela completa germinação, conforme conceito botânico, sendo marcado, em muitos casos, pelo aparecimento da radícula, seguido pelos cotilédones com crescimento no sentido da luz (FENNER e TOMPSON, 2005). Segundo os mesmos autores, os fatores que podem limitar o estabelecimento de plântulas são: luz, água e nutrientes, por meio da competição entre indivíduos; e herbivoria, por animais vertebrados (muitas vezes roedores) e invertebrados (insetos e moluscos).

Conforme Silva et al. (2007), a partir da regeneração natural é possível fazer uma análise efetiva que permita diagnosticar o estado de conservação dos fragmentos e a sua resposta frente às alterações naturais ou antrópicas no ambiente, $\mathrm{o}$ que é importante na medida em que essa regeneração forma um conjunto de indivíduos capazes de serem recrutados para estágios superiores. 
Neste contexto, os mecanismos de regeneração natural são indicativos de como a floresta responderá a uma alteração ambiental, o que será de grande importância na avaliação da possibilidade de utilização do próprio potencial regenerativo da floresta, mediante o emprego de determinados tratamentos silviculturais.

Dessa forma, este estudo teve como objetivo obter informações sobre o potencial dos mecanismos de regeneração da Floresta Estacional Decidual, visando sua conservação e recuperação. Neste sentido procurou-se responder as seguintes questões:

a) Que espécies compõem os diferentes mecanismos de regeneração e quais as suas características autoecológicas?

b) Quais espécies predominam em cada mecanismo e seu potencial para a recuperação e enriquecimento em remanescentes de floresta nativa da região?

\section{MATERIAL E MÉTODOS}

\section{Localização}

$\mathrm{O}$ estudo foi realizado em um remanescente deFloresta Estacional Decidual localizado no Campo

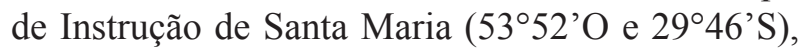
pertencente ao Ministério da Defesa, no município de Santa Maria, RS. A área apresenta 5876 ha e o remanescente estudado, aproximadamente, 560 ha.

As principais classes de solo da região pertencem à Unidade de Mapeamento Santa Maria e são denominadas: Argissolo Bruno-Acinzentado Alítico úmbrico e Argissolo Amarelo Alítico típico, ambas originadas de siltitos e arenito, e que ocorrem em duas situações de paisagem: uma dominando o relevo suavemente ondulado e, outra, ocupando áreas de coxilhas em cotas intermediárias entre Argissolos Vermelhos (Unidade São Pedro) e em cotas mais altas (STRECK et al., 2008).

O clima da região é Cfa, conforme classificação de Köppen, com temperatura média de 17,9 a $19,2^{\circ} \mathrm{C}$, podendo ocorrer geadas de abril a novembro e precipitação média anual entre 1400 e $1760 \mathrm{~mm}$ (LEMOS et al., 1973).

A tipologia florestal da região é caracterizada como Floresta Estacional Decidual, com predominância das famílias Myrtaceae, Lauraceae, Fabaceae, Euphorbiaceae, entre outras (RIO GRANDE DO SUL, 2002). Essa região é caracterizada por ser tipicamente ombrófila, sem período seco e com bastante intensidade e regularidade pluviométrica. No entanto, apresenta dois períodos térmicos bem distintos: um, de 4 a 5 meses, que corresponde à estação mais quente, com temperatura média anual superior a $20^{\circ} \mathrm{C}$, e outra mais fria, com médias anuais inferiores a $15^{\circ} \mathrm{C}$. Neste período frio, mais de $50 \%$ dos indivíduos do estrato superior perdem as suas folhas, o que confere a característica estacional desta tipologia.

\section{Caracterização dos mecanismos de regeneração natural}

Os mecanismos de regeneração natural avaliados neste estudo foram: chuva de sementes, banco de sementes do solo, banco de plântulas e regeneração natural estabelecida, propriamente dita.

Os mecanismos de regeneração natural foram avaliados a partir da demarcação de 14 unidades amostrais de $20 \times 100$ metros $\left(2000 \mathrm{~m}^{2}\right)$, distribuídas de forma sistemática na área a cada 500 $\mathrm{m}$ de distância. As parcelas foram subdivididas em 20 subparcelas de $10 \mathrm{~m} \times 10 \mathrm{~m}$, dentro das quais foram selecionadas, aleatoriamente, 5 subparcelas onde se procedeu ao estudo de cada um dos mecanismos (Figura 1).

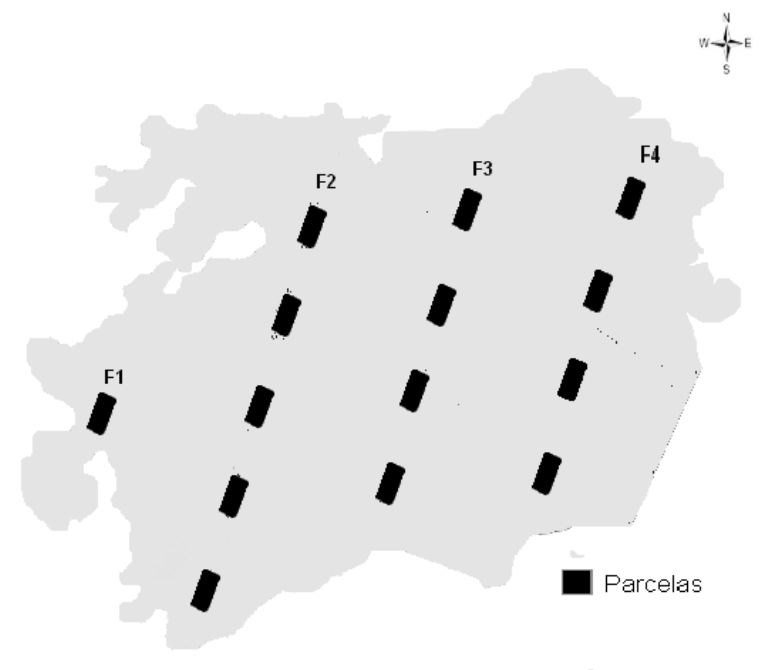

FIGURA 1: Distribuição das parcelas na área e detalhamento da intensidade amostral em remanescente de Floresta Estacional Decidual, CISM, Santa Maria, RS.

FIGURE 1: Distribution of plots in the area and details of sampling intensity on the remaining of Seasonal Deciduous Forest, CISM, Santa Maria, RS state. 


\section{Chuva de sementes}

A chuva de sementes foi observada em 70 coletores de $1 \mathrm{~m}$ x $1 \mathrm{~m}$, revestidos com sombrite $\AA$, instalados a $50 \mathrm{~cm}$ do solo sobre canos de PVC, sendo distribuídos de forma aleatória, totalizando $70 \mathrm{~m}^{2} \mathrm{de}$ área amostrada. As coletas foram feitas mensalmente durante o período de 12 meses, compreendido entre outubro de 2007 e setembro de 2008 . Os propágulos depositados nos coletores foram conduzidos ao Laboratório de Silvicultura do Departamento de Ciências Florestais da UFSM. Todas as sementes com mais de $1 \mathrm{~mm}$ foram separadas da serapilheira, identificadas por meio de bibliografias, observação de especialistas ou observação da plântula após a germinação em viveiro, sendo, então, quantificadas.

\section{Banco de sementes do solo}

O procedimento para avaliação do banco de sementes do solo foi realizado por meio do uso de um gabarito de ferro com dimensões de $25 \mathrm{~cm}$ x 25 $\mathrm{cm}$ que permitiu a coleta do solo a profundidade de $5 \mathrm{~cm}$, sem serapilheira. As coletas foram realizadas dentro das 70 subparcelas utilizadas para o estudo da chuva de sementes, tomando-se sempre os pontos centrais das mesmas.

As amostras foram conduzidas para casa de vegetação, onde o material foi espalhado sobre $5 \mathrm{~cm}$ de vermiculita, em bandeja de plástico ( 40 × 25 x 10 $\mathrm{cm}$ ), para germinação.

Durante sete meses, as amostras receberam irrigação e foram monitoradas diariamente. Após a emergência das sementes, as plântulas foram identificadas e quantificadas semanalmente.

\section{Banco de plântulas e regeneração natural estabelecida}

A regeneração natural considerou indivíduos arbóreos e arbustivos com altura $\geq 30 \mathrm{~cm}$ e DAP (diâmetro a altura do peito) $<5 \mathrm{~cm}$, divididos em duas classes de tamanho:

- Banco de plântulas: indivíduos com altura $\geq 30$ $\mathrm{cm}$ e DAP $<1 \mathrm{~cm}$, avaliados em 70 parcelas de 2 × 2 m;

- Regeneração natural estabelecida: indivíduos com $1 \mathrm{~cm} \leq$ DAP $<5 \mathrm{~cm}$, avaliados em 70 parcelas de 5 × $5 \mathrm{~m}$.

Sendo que o DAP era medido apenas para aqueles indivíduos regenerantes que tinham altura maior que $1,30 \mathrm{~m}$ e DAP menor que $5 \mathrm{~cm}$.

A identificação das plantas foi realizada, sempre que possível, a campo ou por especialistas no herbário do Departamento de Ciências Florestais (HDCF), mediante análise de material vegetativo coletado a campo. Mediu-se altura e diâmetro a altura do peito de todos os indivíduos.

\section{Análise dos dados}

Considerando que os dados não apresentaram distribuição normal, a análise para verificação de ocorrência de diferenças entre os agrupamentos florísticos formados na vegetação adulta Grupo 1 (caracterizado por ambiente em estágio mais avançado de sucessão) e Grupo 2 (caracterizado por floresta em estágio secundário de sucessão) (ALMEIDA et al., 2010) foi realizada através da estatística não paramétrica da soma das ordens Wilcoxon (CARNELUTTI FILHO et al., 2001).

$$
W=\sum O j
$$

Em que:

$W=$ soma das ordens de $\mathrm{Y}_{\mathrm{i}}$

$O_{j}=$ ordem de $\mathrm{Y}_{\mathrm{j}}$ na classificação conjunta de $\mathrm{N}=\mathrm{m}+\mathrm{n}$ observações.

Hipóteses: $\mathrm{H}_{0}: \Delta=0 ; \mathrm{H}_{1} . \Delta \neq 0$.

Rejeita-se $\mathrm{H}_{0}$ se $\mathrm{W} \geq \mathrm{W}_{1-\alpha}$ e $\mathrm{W} \geq \mathrm{W}_{\alpha}$

Os valores dos dados do primeiro grupo $(\mathrm{m})$ e do segundo $(n)$ de W são tabelados e, quando $m$ e $n$ tendem para o infinito ou extrapolam os limites da tabela, calcula-se a estatística $\mathrm{W}^{*}$ e os testes ficam baseados na distribuição normal padrão $\mathrm{W}^{*} \cap \mathrm{N}$ $(0 ; 1)$. A hipótese será $W^{*} \geq Z_{\alpha}$, onde $Z_{\alpha}$ é o limite da distribuição normal padrão ao nível de significância 0,05 .

$$
W *=\frac{W-\left(\frac{n \times(n+m+1)}{2}\right)}{\sqrt{\frac{m \times n(m+n+1)}{12}}}
$$

Em que:

$W^{*}=$ soma das ordens calculado;

$\mathrm{m}=$ número de observações do primeiro grupo;

$\mathrm{n}=$ número de observações do segundo grupo;

$W=$ soma das ordens de $\mathrm{Y}_{\mathrm{j}}$.

Após verificação de diferença entre os agrupamentos, foi calculada a densidade absoluta (DA) (FELFILI e VENTUROLI, 2000) das espécies em cada grupo, se houve diferença significativa entre os eles, ou para o total da floresta, se não houve diferença significativa entre os grupos.

Para banco de plântulas e regeneração natural estabelecida, além da Densidade Absoluta, calculou-se o Índice de diversidade de Shannon 
(H') (FELFILI e VENTUROLI, 2000) e o de equabilidade (J) (ROIZMAN, 1993; ARAUJO, 2002).

\section{RESULTADOS E DISCUSSÕES}

\section{Chuva de sementes}

Durante um ano de observações, 71 espécies procedentes da chuva de sementes foram amostradas. Dentre essas, 44 foram identificadas em nível de espécie, quatro em nível de gênero, uma em nível de família e 22 não foram identificadas (Tabela 1). A dificuldade de identificação ocorre, principalmente, quando o número de sementes na amostra é baixo. Espécies não identificadas foram consideradas como morfoespécies.

As famílias com maior número de espécies foram Myrtaceae (5), Sapindaceae (5), Asteraceae (4), Bignoniaceae, Fabaceae, Meliaceae e Rutaceae, cada uma com três espécies.

TABELA 1: Espécies procedentes da chuva de sementes, do banco de sementes do solo, do banco de plântulas e da regeneração natural estabelecida, em remanescente de Floresta Estacional Decidual, Santa Maria, RS.

TABLE 1: Species from the seed rain, from the soil seed bank, from the seedling bank and from the established natural regeneration in a Seasonal Deciduous Forest fragment, in Santa Maria, RS state.

\begin{tabular}{|c|c|c|c|c|c|c|c|}
\hline Família & Nome científico & Nome comum & $\mathrm{FV}$ & $\mathrm{CS}$ & BSS & $\mathrm{BP}$ & RNE \\
\hline Acanthaceae & Ruelia sp. & - & $\mathrm{H}$ & & & $\mathrm{x}$ & \\
\hline Annonaceae & Rollinia salicifolia Schltdl. & - & $\mathrm{A}$ & $\mathrm{x}$ & & $\mathrm{x}$ & $\mathrm{x}$ \\
\hline Apiaceae & $\begin{array}{l}\text { Hydrocotyle leucocephala Cham. e } \\
\text { Schl. }\end{array}$ & erva-capitão & $\mathrm{H}$ & & $\mathrm{x}$ & & \\
\hline Araliaceae & $\begin{array}{l}\text { Schefflera morototoni (Aubl.) } \\
\text { Maguire, Steyerm. \& Frodin. }\end{array}$ & caixeta & A & $\mathrm{x}$ & $\mathrm{x}$ & & \\
\hline Arecaceae & $\begin{array}{l}\text { Syagrus romanzoffiana (Cham.) } \\
\text { Glassman }\end{array}$ & jerivá & A & $\mathrm{x}$ & & $\mathrm{x}$ & \\
\hline \multirow{15}{*}{ Asteraceae } & Baccharis sp. & - & $\mathrm{H}$ & & $\mathrm{x}$ & & \\
\hline & Chaptalia nutans (L.) Pol. & lingua-de-vaca & $\mathrm{H}$ & $\mathrm{x}$ & & & \\
\hline & Coniza bonariensis (L.) Cronq. & - & $\mathrm{H}$ & & $\mathrm{x}$ & & \\
\hline & $\begin{array}{l}\text { Dasyphyllum spinescens (Less.) } \\
\text { Cabrera }\end{array}$ & açucará & $\mathrm{C}$ & $\mathrm{x}$ & & $\mathrm{x}$ & $\mathrm{x}$ \\
\hline & Elephantopus sp. & pé-de-elefante & $\mathrm{H}$ & & $\mathrm{x}$ & & \\
\hline & $\begin{array}{l}\text { Erechtites valerianifolius (Link ex } \\
\text { Spreng.) DC. }\end{array}$ & caruru & $\mathrm{H}$ & $\mathrm{x}$ & $\mathrm{x}$ & & \\
\hline & Facelis apiculata Cass. & macelinha & $\mathrm{H}$ & & $\mathrm{x}$ & & \\
\hline & Gamochaeta americana (Mill.) Wedd. & - & $\mathrm{H}$ & & $\mathrm{x}$ & & \\
\hline & Gamochaeta sp. & - & $\mathrm{H}$ & & $\mathrm{x}$ & & \\
\hline & $\begin{array}{l}\text { Gamochaeta coarctata (Willd.) } \\
\text { Kerguélen }\end{array}$ & macela & $\mathrm{H}$ & & $\mathrm{x}$ & & \\
\hline & $\begin{array}{l}\text { Macfadyena unguis-cati (L.) A. H. } \\
\text { Gentry }\end{array}$ & cipó-unha-de-gato & $\mathrm{C}$ & $\mathrm{x}$ & & & \\
\hline & Mikania sp. & - & $\mathrm{C}$ & & $\mathrm{x}$ & & \\
\hline & Mikania micrantha Kunth & mikania & AR & & $\mathrm{x}$ & & \\
\hline & Parthenium cesterapharus & losna-do-campo & $\mathrm{H}$ & & $\mathrm{x}$ & & \\
\hline & $\begin{array}{l}\text { Podocoma notobellidiastrum (Griseb.) } \\
\text { G. L. Nesom }\end{array}$ & - & $\mathrm{H}$ & & $\mathrm{x}$ & & \\
\hline \multirow{3}{*}{ Asteraceae } & Senecio brasiliensis (Spreng.) Less. & maria-mole & $\mathrm{H}$ & & $\mathrm{x}$ & & \\
\hline & Senecio oxiphyllus DC. & maria-mole & $\mathrm{H}$ & & $\mathrm{x}$ & & \\
\hline & Spathicarpa hastifolia Hook. & - & $\mathrm{H}$ & $\mathrm{x}$ & & & \\
\hline Begoniaceae & Begonia sp. & begônia & $\mathrm{H}$ & & $\mathrm{x}$ & & \\
\hline
\end{tabular}


TABELA 1: Continuação ...

TABLE 1: Continued ..

\begin{tabular}{|c|c|c|c|c|c|c|c|}
\hline Família & Nome científico & Nome comum & FV & $\mathrm{CS}$ & BSS & $\mathrm{BP}$ & RNE \\
\hline \multirow{2}{*}{ Bignoniaceae } & Tabebuia heptaphylla (Vell.) Toledo & ipê-roxo & Á & $\mathrm{x}$ & & & \\
\hline & Tecoma stans (L.) Juss. ex Kunth & ipê-de-jardim & $\mathrm{H}$ & & $\mathrm{x}$ & & \\
\hline Brassicaceae & Lepidium sp. & mastruz & $\mathrm{H}$ & & $\mathrm{x}$ & & \\
\hline Callitrichaceae & Callitriche sp. & - & $\mathrm{H}$ & & $\mathrm{x}$ & & \\
\hline Caryophyllaceae & $\begin{array}{l}\text { Drymaria cordata (L.) Willd. ex } \\
\text { Schult. }\end{array}$ & cordão-de-sapo & $\mathrm{C}$ & & $\mathrm{x}$ & & \\
\hline Combretaceae & Combretum leprosum Mart. & carne-de-vaca & $\mathrm{AR}$ & $\mathrm{x}$ & & & \\
\hline \multirow{2}{*}{ Commelinaceae } & Commelina robusta Kunth & trapoeiraba & $\mathrm{H}$ & & $\mathrm{x}$ & & \\
\hline & Commelina virginica $\mathrm{L}$. & trapoeiraba & $\mathrm{H}$ & & $\mathrm{x}$ & & \\
\hline Convolvulaceae & $\begin{array}{l}\text { Dichondra repens J.R. Forst. \& G. } \\
\text { Forst. }\end{array}$ & orelha-de-rato & $\mathrm{H}$ & & $\mathrm{x}$ & & \\
\hline Cucurbitaceae & Cucurbitaceae NI7 & - & $\mathrm{H}$ & & $\mathrm{x}$ & & \\
\hline Cyperaceae & Bulbostylis sp. & cabelo-de-porco & $\mathrm{H}$ & & $\mathrm{x}$ & & \\
\hline \multirow{2}{*}{ Dioscoreaceae } & Dioscorea sp. & jarrinha & $\mathrm{H}$ & & $\mathrm{x}$ & & \\
\hline & Dioscorea sp.1 & - & $\mathrm{H}$ & & $\mathrm{x}$ & & \\
\hline Erythroxylaceae & $\begin{array}{l}\text { Erythroxylum argentinum O. E. } \\
\text { Schulz }\end{array}$ & cocão & AR & $\mathrm{x}$ & & & \\
\hline \multirow{4}{*}{ Euphorbiaceae } & Chamaesyce sp. & & $\mathrm{H}$ & & $\mathrm{x}$ & & \\
\hline & $\begin{array}{l}\text { Gymnanthes concolor (Spreng.) Müll. } \\
\text { Arg. }\end{array}$ & laranjeira-do-mato & AR & $\mathrm{x}$ & $\mathrm{x}$ & $\mathrm{x}$ & $\mathrm{x}$ \\
\hline & Phylantus tenellus Roxb. & quebra-pedra & $\mathrm{H}$ & & $\mathrm{x}$ & & \\
\hline & $\begin{array}{l}\text { Sebastiania commersoniana (Baill.) } \\
\text { L.B. Sm. \& Downs }\end{array}$ & branquilho-comum & A & $\mathrm{x}$ & $\mathrm{x}$ & $\mathrm{x}$ & \\
\hline \multirow{5}{*}{ Fabaceae } & Desmodium incanum DC. & pega-pega & $\mathrm{H}$ & & $\mathrm{x}$ & & \\
\hline & $\begin{array}{l}\text { Enterolobium contortisiliquum (Vell.) } \\
\text { Morong }\end{array}$ & timbaúva & A & $\mathrm{x}$ & & & \\
\hline & Mimosa bimucronata (DC.) Kuntze & maricá & AR & $\mathrm{x}$ & & $\mathrm{x}$ & \\
\hline & Myrocarpus frondosus Allemão & cabriúva & A & & & $\mathrm{x}$ & $\mathrm{x}$ \\
\hline & $\begin{array}{l}\text { Parapiptadenia rigida (Benth.) } \\
\text { Brenan }\end{array}$ & angico-vermelho & A & $\mathrm{x}$ & & $\mathrm{x}$ & $\mathrm{x}$ \\
\hline \multirow{4}{*}{ Flacourtiaceae } & Banara tomentosa Clos & guaçatunga-branca & A & & & $\mathrm{x}$ & $\mathrm{x}$ \\
\hline & Casearia decandra Jacq. & guaçatunga & A & $\mathrm{x}$ & & & $\mathrm{x}$ \\
\hline & Casearia sylvestris $\mathrm{Sw}$. & carvalhinho & A & & & $\mathrm{x}$ & $\mathrm{x}$ \\
\hline & Xylosma pseudosalzmanii Sleumer & espinho-judeo & AR & & & $\mathrm{x}$ & \\
\hline \multirow{2}{*}{ Hippocrateaceae } & Anthodon sp. & - & $\mathrm{C}$ & $\mathrm{x}$ & & & \\
\hline & Pristimera andina Miers & cipó-pau & AR & & & $\mathrm{x}$ & $\mathrm{x}$ \\
\hline Lamiaceae & Stachys arvensis L. & hortelão-da-roça & $\mathrm{H}$ & & $\mathrm{x}$ & & \\
\hline \multirow[t]{2}{*}{ Lauraceae } & $\begin{array}{l}\text { Nectandra megapotamica (Spreng.) } \\
\text { Mez }\end{array}$ & canela-amarela & A & $\mathrm{x}$ & & $\mathrm{x}$ & $\mathrm{x}$ \\
\hline & Ocotea sp. & canela & A & & & $\mathrm{x}$ & \\
\hline Loasaceae & Blumenbachia urens Urb. & - & $\mathrm{H}$ & & $\mathrm{x}$ & & \\
\hline Loranthaceae & $\begin{array}{l}\text { Struthanthus flexicaulis (Mart. ex } \\
\text { Schult. F.) Mart. }\end{array}$ & erva-de-passarinho & $\mathrm{C}$ & $\mathrm{x}$ & & & \\
\hline Lythraceae & Heimia myrtifolia Cham. \& Schltdl. & - & $\mathrm{H}$ & & $\mathrm{x}$ & & \\
\hline Melastomataceae & Miconia sp. & quaresmeira & AR & & $\mathrm{x}$ & $\mathrm{x}$ & $\mathrm{x}$ \\
\hline \multirow{4}{*}{ Meliaceae } & Cedrela fissilis Vell. & cedro & $\mathrm{A}$ & $\mathrm{x}$ & & $\mathrm{x}$ & \\
\hline & Trichilia catigua A. Juss. & catiguá-verdadeiro & A & & & $\mathrm{x}$ & $\mathrm{x}$ \\
\hline & Trichilia claussenii C. DC. & catiguá-vermelho & A & $\mathrm{x}$ & & $\mathrm{x}$ & $\mathrm{x}$ \\
\hline & Trichilia elegans A. Juss. & catiguá-de-ervilha & A & $\mathrm{x}$ & & $\mathrm{x}$ & $\mathrm{x}$ \\
\hline
\end{tabular}


TABELA 1: Continuação ...

TABLE 1: Continued ..

\begin{tabular}{|c|c|c|c|c|c|c|c|}
\hline Família & Nome científico & Nome comum & $\mathrm{FV}$ & $\mathrm{CS}$ & BSS & $\mathrm{BP}$ & RNE \\
\hline Menispermaceae & Abuta sp. & - & $\mathrm{C}$ & $\mathrm{x}$ & & & \\
\hline \multirow[b]{2}{*}{ Moraceae } & Ficus luschnathiana (Miq.) Miq. & Figueira-mata-pau & A & $\mathrm{x}$ & $\mathrm{x}$ & & \\
\hline & $\begin{array}{l}\text { Sorocea bonplandii (Baill.) W.C. } \\
\text { Burger, Lanj. \& Wess. Boer }\end{array}$ & cincho & AR & & & $\mathrm{x}$ & $\mathrm{x}$ \\
\hline \multirow{3}{*}{ Myrsinaceae } & $\begin{array}{l}\text { Myrsine coriacea (Sw.) R. Br. ex } \\
\text { Roem. \& Schult. }\end{array}$ & capororoquinha & A & & & $\mathrm{x}$ & \\
\hline & Myrsine sp. & - & A & & & $\mathrm{x}$ & \\
\hline & Rapanea umbellata (Mart.) Mez & capororoca & AR & $\mathrm{x}$ & & & \\
\hline \multirow{8}{*}{ Myrtaceae } & Campomanesia xanthocarpa O.Berg & guabiroba & $\mathrm{A}$ & $\mathrm{x}$ & & $\mathrm{x}$ & $\mathrm{x}$ \\
\hline & Eugenia involucrata DC. & cerejeira & $\mathrm{A}$ & $\mathrm{x}$ & & $\mathrm{x}$ & $\mathrm{x}$ \\
\hline & Eugenia rostrifolia D. Legrand & batinga & A & $\mathrm{x}$ & & $\mathrm{x}$ & $\mathrm{x}$ \\
\hline & Eugenia uniflora L. & pitangueira & A & $\mathrm{x}$ & & $\mathrm{x}$ & $\mathrm{x}$ \\
\hline & Eugenia uruguayensis Cambess. & guamirim & A & & & $\mathrm{x}$ & \\
\hline & $\begin{array}{l}\text { Myrcianthes pungens (O. Berg) D. } \\
\text { Legrand }\end{array}$ & guabijú & A & & & $\mathrm{x}$ & $\mathrm{x}$ \\
\hline & Plinia rivularis (Cambess.) Rotman & guaporiti & A & $\mathrm{x}$ & & $\mathrm{x}$ & $\mathrm{x}$ \\
\hline & Psidium guajava L. & goiabeira & A & & $\mathrm{x}$ & & \\
\hline Onagraceae & Onagraceae NI69 & - & $\mathrm{H}$ & & $\mathrm{x}$ & & \\
\hline Oxalidaceae & Oxalis refracta A.St.-Hil. & falso-trevo & $\mathrm{H}$ & & $\mathrm{x}$ & & \\
\hline \multirow{2}{*}{ Phytolccaceae } & Phytolacca dioica $\mathrm{L}$. & umbú & A & $\mathrm{x}$ & $\mathrm{x}$ & & \\
\hline & Seguiera sp. & cipó-umbú & AR & $\mathrm{x}$ & & & \\
\hline \multirow{2}{*}{ Piperaceae } & Piper aduncum L. & - & AR & & & $\mathrm{x}$ & $\mathrm{x}$ \\
\hline & Piper mikanianum (Kunth) Steud. & pariparoba & AR & & $\mathrm{x}$ & & \\
\hline \multirow{2}{*}{ Poaceae } & Chusquea sp. & criciúma & $\mathrm{H}$ & $\mathrm{x}$ & & $\mathrm{x}$ & $\mathrm{x}$ \\
\hline & Poaceae NI76 & - & $\mathrm{H}$ & & $\mathrm{x}$ & & \\
\hline Polygonaceae & Ruprechtia laxiflora Meisn. & marmeleiro-do-mato & AR & $\mathrm{x}$ & & $\mathrm{x}$ & \\
\hline Portulacaceae & Talinum paniculatum (Jacq.) Gaertn. & maria-gorda & $\mathrm{H}$ & & $\mathrm{x}$ & & \\
\hline \multirow{3}{*}{ Rosaceae } & Citrus sp. & laranjeira & A & $\mathrm{x}$ & & $\mathrm{x}$ & \\
\hline & Prunus myrtifolia (L.) Urb. & pessegueiro-do-mato & AR & $\mathrm{x}$ & & $\mathrm{x}$ & \\
\hline & Rubus brasiliensis Mart. & amora-do-mato & AR & & $\mathrm{x}$ & $\mathrm{x}$ & \\
\hline \multirow{2}{*}{ Rubiaceae } & Chomelia obtusa Cham. \& Schltdl. & viuvinha & $\mathrm{AR}$ & & & $\mathrm{x}$ & \\
\hline & Rubiaceae NI74 & - & $\mathrm{H}$ & & $\mathrm{x}$ & & \\
\hline \multirow{3}{*}{ Rutaceae } & Helietta apiculata Benth. & canela-de-veado & A & $\mathrm{x}$ & & $\mathrm{x}$ & \\
\hline & Pilocarpus pennatifolius Lem. & baga-de-macaco & AR & $\mathrm{x}$ & & $\mathrm{x}$ & $\mathrm{x}$ \\
\hline & Zantoxilum fagara (L.) Sarg. & coentrilho & A & & & & $\mathrm{x}$ \\
\hline Rutaceae & Zanthoxylum rhoifolium Lam. & mamica-de-cadela & $\mathrm{A}$ & & $\mathrm{x}$ & & \\
\hline \multirow{6}{*}{ Sapindaceae } & $\begin{array}{l}\text { Allophylus edulis (A. St.-Hil., } \\
\text { Cambess. \& A. Juss.) Radlk. }\end{array}$ & chal-chal & A & $\mathrm{x}$ & & $\mathrm{x}$ & \\
\hline & Allophylus guaraniticus Radlk. & vacum & A & & & $\mathrm{x}$ & $\mathrm{x}$ \\
\hline & Cupania vernalis Cambess. & camboatá-vermelho & A & $\mathrm{x}$ & & $\mathrm{x}$ & \\
\hline & Matayba elaeagnoides Radlk. & Camboatá-branco & $\mathrm{A}$ & $\mathrm{x}$ & & $\mathrm{x}$ & \\
\hline & Serjania sp. & - & $\mathrm{C}$ & $\mathrm{x}$ & $\mathrm{x}$ & & \\
\hline & Urvillaea $\mathrm{sp}$. & - & $\mathrm{C}$ & $\mathrm{x}$ & & & \\
\hline
\end{tabular}

Continua ... 
TABELA 1: Continuação ...

TABLE 1: Continued ..

\begin{tabular}{|c|c|c|c|c|c|c|c|}
\hline Família & Nome científico & Nome comum & $\mathrm{FV}$ & $\mathrm{CS}$ & BSS & $\mathrm{BP}$ & RNE \\
\hline \multirow{3}{*}{ Sapotaceae } & $\begin{array}{l}\text { Chrysophyllum gonocarpum (Mart. } \\
\text { \& Eichler ex Miq.) Engl. }\end{array}$ & aguaí-da-serra & A & $\mathrm{x}$ & & $\mathrm{x}$ & \\
\hline & $\begin{array}{l}\text { Chrysophyllum marginatum (Hook. } \\
\text { \& Arn.) Radlk. }\end{array}$ & aguaí-leitero & A & $\mathrm{x}$ & & $\mathrm{x}$ & $\mathrm{x}$ \\
\hline & $\begin{array}{l}\text { Sidoroxylon obtusifolium (Roem. \& } \\
\text { Schult.) Penning }\end{array}$ & sombra-de-touro & AR & & & & $\mathrm{x}$ \\
\hline \multirow{7}{*}{ Solanaceae } & Brunfelsia uniflora (Pohl) D. Don & primavera & $\mathrm{AR}$ & & & & $\mathrm{x}$ \\
\hline & Cestrum sp. & coerana & $\mathrm{H}$ & & $\mathrm{x}$ & & \\
\hline & Physalis pubescens L. & joá-de-capote & $\mathrm{H}$ & & $\mathrm{x}$ & & \\
\hline & Solanum aculeatissimum Jacq. & joá-de-espinho & $\mathrm{H}$ & & $\mathrm{x}$ & & \\
\hline & Solanum mauritianum Scop. & fumo-bravo & $\mathrm{H}$ & & $\mathrm{x}$ & & \\
\hline & Solanum pseudioquina Saint-Hilaire & peloteiro & AR & & & & $\mathrm{x}$ \\
\hline & Solanun americanum Mill. & maria-pretinha & $\mathrm{AR}$ & & $\mathrm{x}$ & & \\
\hline Sterculiaceae & Byttneria urticifolia $\mathrm{K}$. Schum. & cida & $\mathrm{H}$ & & $\mathrm{x}$ & & \\
\hline Symplocaceae & Symplocus uniflora (Pohl) Bent. & sete-sangrias & $\mathrm{AR}$ & & & & $\mathrm{x}$ \\
\hline Tiliaceae & Luehea divaricata Mart. & açoita-cavalo & A & $\mathrm{x}$ & $\mathrm{x}$ & $\mathrm{x}$ & \\
\hline \multirow{2}{*}{ Ulmaceae } & Celtis iguanaea (Jacq.) Sarg. & esporão-de-galo & $\mathrm{AR}$ & $\mathrm{x}$ & $\mathrm{x}$ & $\mathrm{x}$ & $\mathrm{x}$ \\
\hline & Trema micrantha (L.) Blume & grandiúva & AR & & $\mathrm{x}$ & & \\
\hline \multirow{4}{*}{ Urticaceae } & Boehmeria sp. & - & $\mathrm{H}$ & & $\mathrm{x}$ & & \\
\hline & $\begin{array}{l}\text { Urera baccifera (L.) Gaudich. ex } \\
\text { Wedd. }\end{array}$ & urtigão & AR & & $\mathrm{x}$ & & \\
\hline & Urtica dioica $\mathrm{L}$. & - & $\mathrm{H}$ & & $\mathrm{x}$ & & \\
\hline & Urticaceae NI129 & - & $\mathrm{H}$ & & $\mathrm{x}$ & & \\
\hline Verbanaceae & $\begin{array}{l}\text { Vitex megapotamica (Spreng.) } \\
\text { Moldenke }\end{array}$ & tarumã & A & & & & $\mathrm{x}$ \\
\hline \multirow[b]{2}{*}{ Violaceae } & Anchietea parvifolia Hallier f. & cipó-suma & $\mathrm{C}$ & $\mathrm{x}$ & & & \\
\hline & $\begin{array}{l}\text { Hybanthus bigibbosus (A. St.-Hil.) } \\
\text { Hassl. }\end{array}$ & viuvinha & AR & & $\mathrm{x}$ & $\mathrm{x}$ & $\mathrm{x}$ \\
\hline
\end{tabular}

Em que: $\mathrm{FV}=$ forma de vida; $\mathrm{AR}=$ arbusto; $\mathrm{A}=$ árvore, $\mathrm{CS}=$ chuva de sementes; $\mathrm{BSS}=$ banco de sementes do solo; $\mathrm{BP}=$ banco de plântulas; $\mathrm{RNE}=$ regeneração natural estabelecida; $\mathrm{H}=$ altura; $\mathrm{C}=$ clímax.

O período com maior produção de sementes foi de outubro a dezembro de 2007 e de agosto a setembro de 2008, ou seja, final do inverno e início da primavera, enquanto que a menor produção ocorreu de março a julho de 2008 (Figura 2).

A menor produção, ocorrida de março a julho, pode estar relacionada a fatores climáticos, uma vez que esse período coincide com as menores temperaturas do ano e com o menor comprimento do dia na região, além disso, é quando mais de $50 \%$ do estrato dominante da vegetação adulta perde as suas folhas. As baixas temperaturas e a pouca intensidade luminosa reduzem o processo fotossintético das plantas e, assim, a translocação de fotossintatos para o processo reprodutivo (LARKER, 2000).

Por outro lado, no mês de agosto, que apresenta ainda baixas temperaturas e o menor comprimento do dia, obteve-se maior número de sementes em relação aos demais meses de observação. Esse fato teve contribuição da elevada produção de Chusquea sp. com 389 sementes.m ${ }^{-2}$ em agosto e 247 sementes. $\mathrm{m}^{-2}$ em setembro.

Chusquea sp. é uma espécie conhecida vulgarmente por bambu ou criciúma. Segundo Crouzet (1998) as espécies de bambu possuem inflorescências muito raras e podem permanecer décadas sem produção. Quando essa ocorre, a planta deposita muita energia para floração e para frutificação como uma forma de garantir a sua perpetuação, pois, após este evento, ocorre a morte dos indivíduos adultos.

Tal fato ocorreu na área de estudo, pois a população de Chusquea sp. morreu após a frutificação, dando início a substituição de indivíduos pela formação do banco de plântulas, favorecido pela elevada densidade de sementes dispersadas.

De acordo com o teste Wilcoxon, a chuva 
de sementes mostrou o mesmo comportamento em termos de densidade $(\mathrm{p}<0,05)$ para os dois agrupamentos florísticos observados na vegetação adulta (ALMEIDA et al., 2009), sendo o valor calculado $(0,43)$ menor que o tabelado $(1,96)$, o que indica similaridade na dispersão de sementes na área de estudo.

A densidade média de sementes, aparentemente, viáveis encontradas no remanescente foi de 1350 sementes $\mathrm{m}^{-2}$. Outros estudos mostraram valores menores de densidade, como o de Chami (2008) que indicou 1158 sementes $\mathrm{m}^{-2}$ em Floresta Ombrófila Mista no Rio Grande do Sul. Vieira (1996) verificou 514 sementes $\mathrm{m}^{-2} \mathrm{em}$ floresta de 20 anos e 220 sementes $\mathrm{m}^{-2} \mathrm{em}$ floresta madura na Amazônia. Por outro lado, Rudge (2008) encontrou maior valor (2295 sementes $\mathrm{m}^{-2}$ ) em Floresta Ombrófila Densa no Rio de Janeiro.

A elevada densidade de sementes observada no remanescente foi determinada, principalmente, pelas espécies Chusquea sp. (635 sementes. $\mathrm{m}^{-2}$ ), Dasyphyllum spinescens (247 sementes. $\left.\mathrm{m}^{-2}\right)$, uma espécie da família Asteraceae (151 sementes. $\mathrm{m}^{-2}$ ) que não foi possível identificação e, Gymnanthes concolor (118 sementes. $\left.\mathrm{m}^{-2}\right)$.

Das espécies identificadas, a principal síndrome de dispersão foi zoocórica (48\%), seguida de anemocórica $(40 \%)$ e autocórica (12\%), sendo comum a presença de sementes nas fezes de animais durante as observações.

No remanescente foi observado que a dispersão zoocórica pode ser importante no aumento da riqueza de espécies e na troca de carga genética, além de favorecer o estabelecimento de plântulas, pois Pizo (2003), estudando a dispersão de sementes e a sobrevivência de plântulas de duas espécies de Myrtaceae, constatou que a dispersão aleatória de sementes proporcionada pela avifauna favoreceu o desenvolvimento dos indivíduos jovens destas espécies.

\section{Banco de sementes do solo}

$\mathrm{Na}$ composição florística do banco de sementes foram observadas 108 espécies, das quais 46 foram identificados em nível de espécie, 18 em nível de gênero, 9 em nível de família e 35 não foram identificadas (Tabela 1). As famílias com maior número de espécies foram Asteraceae (18), Solanaceae (5), Euphorbiaceae (4) e Melastomataceae (3). O grande número de espécies não identificadas no banco de sementes do solo está associado, principalmente, a elevada quantidade de espécies herbáceas com morfologia semelhante.

Das espécies observadas no banco de sementes, $74 \%$ são herbáceas, $10 \%$ arbustivas, $9 \%$ arbóreas e $7 \%$ não foi possível definir a forma de vida. Dentre as espécies arbóreas e arbustivas, $43 \%$ são pioneiras, $33 \%$ secundárias inicial, $19 \%$ secundária tardia e 5\% clímax. A predominância de espécies herbáceas e pioneiras é comum em estudos de banco de sementes do solo. Isso ocorre, provavelmente, devido à intensa capacidade reprodutiva dessas espécies, ao pequeno tamanho das sementes e ao seu tegumento duro, muitas vezes tolerantes a maior perda d'água, o que possibilita que se mantenham viáveis por mais tempo no solo (BUDOWISK, 1965; MELO, 2004).

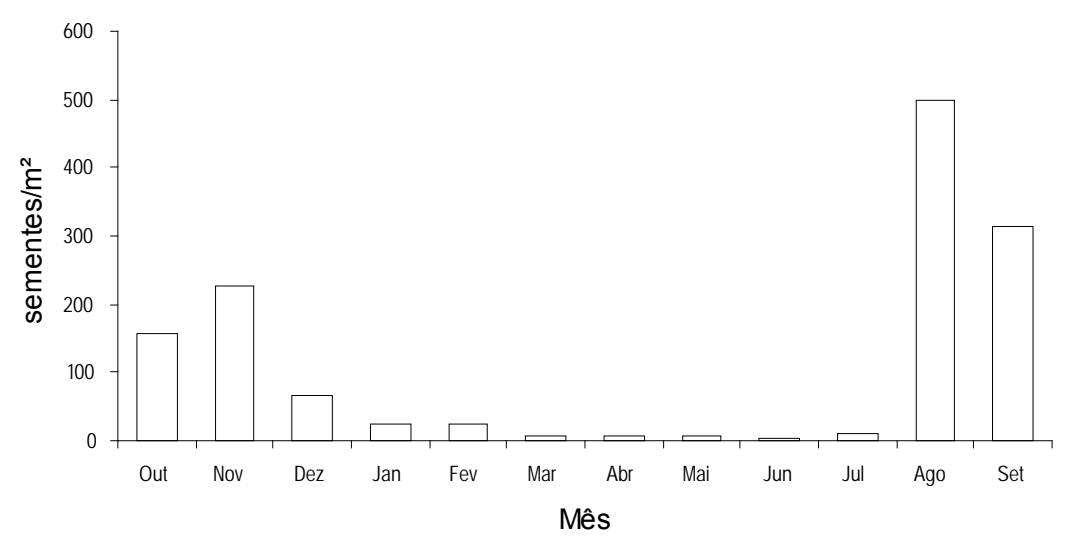

FIGURA 2: Densidade de sementes por $\mathrm{m}^{2}$, dispersadas durante 12 meses de avaliação em remanescente de Floresta Estacional Decidual, Santa Maria, RS.

FIGURE 2: Density of seeds for square meters, scattered over 12 months in the evaluation of a Seasonal Deciduous Forest fragment, in Santa Maria, RS state. 
O banco de sementes do solo mostrou comportamento diferente nos dois agrupamentos florísticos formados na vegetação adulta (W $\left.(2,4)>\mathrm{W}_{\text {tabelado }}(1,96)\right)$. Desta forma, a densidade absoluta foi calculada de acordo com a área total representada por cada agrupamento, sendo o Grupo 1 (típica Floresta Estacional Decidual) representado por 34 parcelas $\left(2,125 \mathrm{~m}^{2}\right)$ e o Grupo 2 (Floresta em estagio secundário de sucessão) por 36 parcelas $\left(2,25 \mathrm{~m}^{2}\right)$.

No Grupo 1 foram observadas 853 sementes.m ${ }^{-2}$, sendo Ficus luschnathiana $\left(68,24\right.$ sementes $\left.\mathrm{m}^{-2}\right)$, Phytolacca dioica $(54,12$ sementes. $\left.\mathrm{m}^{-2}\right)$ e Solanum mauritianum (18,35 sementes. $\mathrm{m}^{-2}$ ) as espécies predominantes.

O Grupo 2 contou com 817 sementes $\mathrm{m}^{-2}$, sendo predominantes as espécies Ficus luschnathiana (42,67 sementes. $\left.\mathrm{m}^{-2}\right)$, Miconia sp. $\left(32,89\right.$ sementes. $\left.^{-2}\right)$, Phytolacca dioica $(32,89$ sementes. $\left.\mathrm{m}^{-2}\right)$ e, Solanum mauritianum $(23,11$ sementes. $\mathrm{m}^{-2}$ ).

As espécies Miconia sp., Solanum mauritianum e Phytolaca dioica são pioneiras, apresentam dispersão zoocórica, possuem sementes pequenas (em torno de $1-2 \mathrm{~mm}$ ) e tolerantes ao dessecamento (BACKES e IRGANG, 2002; LORENZI, 1991 e 2002) o que possivelmente favorece sua presença no banco de sementes do solo.

Ficus luschnathiana é uma espécie que possui frutos zoocóricos e as sementes depositadas em locais altos (árvores, pedras) germinam escassamente (BACKES e IRGANG, 2002). Na área de estudo observou-se grande produção de frutos desta espécie o que pode ter favorecido a sua presença no banco de sementes do solo. Apesar de alguns autores, como Backes e Irgang (2002), caracterizarem como espécie de comportamento clímax, não foi observado sua presença no banco de plântulas.

As espécies observadas no banco de sementes mostram potencial para colonizar áreas alteradas, além de serem atrativas para fauna, o que será importante para a dispersão de outras espécies, uma vez que a riqueza florística em termos de espécies arbóreas e arbustivas é baixa no banco de sementes do solo.

\section{Banco de plântulas e regeneração natural estabelecida}

No banco de plântulas (altura $\geq 30 \mathrm{~cm}$ e DAP $<1 \mathrm{~cm}$ ) foram identificadas 45 espécies, 37 gêneros, 25 famílias e 3 não foram identificadas e, na regeneração natural estabelecida $(1 \mathrm{~cm} \leq \mathrm{DAP}<$ $5 \mathrm{~cm}$ ) foram amostrados 36 espécies, 31 gêneros, 22 famílias e 1 não identificada.

As famílias com maior número de espécies no banco de plântulas foram Myrtaceae, Meliaceae, Sapindaceae e Rutaceae, enquanto na regeneração natural estabelecida as espécies que se destacaram foram Flacourtiaceae, Myrtaceae, Sapotaceae e Solanacea, que tiveram maior destaque. A família Myrtaceae também foi representativa no inventário florestal contínuo do Estado para Floresta Estacional Decidual e em outros trabalhos realizados na região (FARIA et al., 1994; RIO GRANDE DO SUL, 2002; ARAUJO, et al., 2004).

O índice de diversidade de Shannon foi de 2,38 para banco de plântulas e 1,22 para regeneração natural estabelecida, para a equabilidade obtiveramse os valores de 0,61 e 0,34 , respectivamente. Os índices de diversidade indicam baixa diversidade florística do remanescente, ocorrendo predomínio de poucas espécies em ambos os casos, mas, principalmente, na regeneração natural estabelecida. Esse fato sugere que poucas espécies estão realmente se estabelecendo, mesmo tendo ingressado na fase inicial (banco de plântulas). Araujo (2002) descreveu que o predomínio de poucas espécies na vegetação resulta em valores baixos de equabilidade e, consequentemente, baixo índice de diversidade.

A densidade absoluta de indivíduos em ambas as classes foi de 24.961 indivíduos.ha ${ }^{-1}$ (ind. ha $^{-1}$ ) sendo 20.607 para banco de plântulas e 4.354 ind.ha ${ }^{-1}$ para regeneração natural estabelecida, com base nas 70 unidades amostrais.

A partir desses valores, pode-se observar maior dinâmica de recrutamento e mortalidade nas classes de menores tamanhos, demonstrando a tendência natural "J invertido", onde muitas sementes germinam permitindo que indivíduos ingressem no banco de plântulas, mas que uma proporção consideravelmente menor consegue atingir as classes de maior de tamanho (Figura 3).

Resultado semelhante foi encontrado por Araujo et al. (2004) em Floresta Estacional Decidual Ripária, onde $67 \%$ dos indivíduos amostrados na regeneração eram representados por plantas com altura menor que $50 \mathrm{~cm}$ e Chami (2008) que observou, aproximadamente, $55 \%$ dos indivíduos amostrados com essa mesma altura em Floresta Ombrófila Mista. Na área de estudo, essa classe de tamanho representou $67 \%$ dos indivíduos amostrados.

$\mathrm{O}$ número de indivíduos do banco de plântulas no Grupo 1 quando comparado pelo teste

Ci. F1., v. 21, n. 3, jul.-set., 2011 


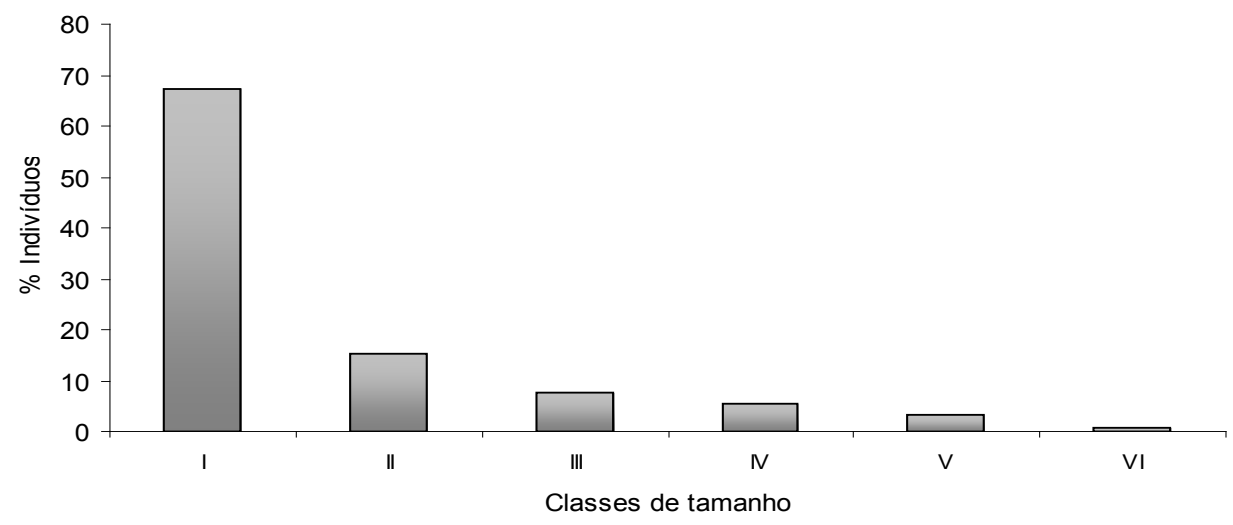

FIGURA 3: Distribuição dos indivíduos no banco de plântulas e regeneração natural estabelecida por classe de tamanho em remanescente de Floresta Estacional Decidual, Santa Maria, RS. (Classe I $(30 \geq \mathrm{h}<100 \mathrm{~cm})$; II $(100 \geq \mathrm{h}$ e DAP $<1 \mathrm{~cm})$, III $(1 \geq \mathrm{DAP}<2 \mathrm{~cm})$; IV $(2 \geq \mathrm{DAP}<3 \mathrm{~cm}), \mathrm{V}(3 \geq$ $\mathrm{DAP}<4 \mathrm{~cm})$; VI $(4 \geq \mathrm{DAP}<5 \mathrm{~cm}))$

FIGURE 3: Distribution of subjects in the seedling bank and natural regeneration established by size class in a Seasonal Deciduous Forest fragment, in Santa Maria, RS state.

de Wilcoxon com o Grupo 2, não indicou diferença entre os diferentes agrupamentos florísticos da vegetação adulta $\left(\mathrm{W}_{\text {calculado }}(0,53)<\mathrm{W}_{\text {tabelado }}(1,96)\right)$. Assim, a densidade absoluta foi calculada com base em um único grupo representado pelas 70 unidades amostrais $(0,028 \mathrm{ha})$. No entanto, na regeneração natural estabelecida, verificou-se diferença significativa para o número de plantas encontradas entre o Grupo 1 (4.941 ind.ha-1 ${ }^{-1}$ ) e Grupo 2 (3.800 ind.ha $\left.{ }^{-1}\right)\left(\mathrm{W}_{\text {calculado }}(2,31)>\mathrm{W}_{\text {tabelado }}(1,96)\right)$, sendo a densidade absoluta calculada de acordo com a área total representada por cada agrupamento, Grupo 1 representado por 34 parcelas ( 0,085 ha) e o Grupo 2 por 36 parcelas $(0,09 \mathrm{ha})$.

No banco de plântulas observou-se a predominância de espécies esciófilas, como Gymnanthes concolor (10.000 ind.ha $\left.{ }^{-1}\right)$ e Sorocea bonplandii (1.036) (REITZ et al., 1988; LORENZI, 2002), e espécies heliófilas, como Eugenia rostrifolia (1.036), Myrocarpus frondosus (821), Parapiptadenia rigida (714), Syagrus romanzoffiana (714), Cupania vernalis (679) e Nectandra megapotamica (642) (LORENZI, 2002). Provavelmente, a abertura do dossel será necessária para o desenvolvimento das espécies heliófilas encontradas no banco de plântulas, enquanto as espécies esciófilas conseguem se desenvolver sob o dossel da floresta, em ambientes mais sombreados, passando para classes de tamanhos maiores.

$\mathrm{Na}$ regeneração natural estabelecida a riqueza florística encontrada no Grupo 1 foi de 20 espécies, distribuídas em 17 gêneros e 15 famílias, enquanto no Grupo 2 foram observadas 31 espécies, pertencentes a 25 gêneros e 19 famílias, além de 1 espécie não identificada.

No Grupo 1 observou-se predomínio de espécies de sombra, o que indica ocorrência de um ambiente em estágio sucessional mais avançado, com a presença de Gymnanthes concolor (4.177 ind.ha $\left.{ }^{-1}\right)$, Trichilia elegans (141 ind.ha $\left.{ }^{-1}\right)$, Sorocea bomplandii (105 ind.ha ${ }^{-1}$ ) e Trichilia claussenii (94 ind.ha ${ }^{-1}$ )

O Grupo 2 observou-se o predomínio das espécies Gymnanthes concolor (2.733 ind.ha $\left.{ }^{-1}\right)$, Eugenia rostrifolia (178 ind.ha $\left.{ }^{-1}\right)$, Dasyphyllum spinecens (133 ind.ha $\left.{ }^{-1}\right)$, Brunfelsia uniflora (89 ind. ha $\left.^{-1}\right)$ e Sorocea bonplandii (89 ind.ha-1 ${ }^{-1}$.

\section{Os mecanismos de regeneração natural}

A presença de indivíduos nos diferentes mecanismos de regeneração, representados por diásporos dispersos, germinados e estabelecidos, está associada com a autoecologia das espécies, que se relaciona à demanda por luz e à forma de dispersão.

Na chuva de sementes, banco de plântulas e regeneração natural estabelecida foi possível observar abundância das espécies Gymnanthes concolor e Dasyphyllum spinescens, as quais apresentam, respectivamente, características de secundária tardia (REITZ et al., 1988) e clímax (RIO GRANDE DO SUL, 2007), apresentando, desta forma, potencial para se manterem na área a partir desses mecanismos. 
Cupania vernalis, Sebastiania commersoniana e Syagrus romanzoffiana estiveram bem representadas na chuva de sementes e banco de plântulas. Essas espécies são heliófilas (LORENZI, 2002), logo, os indivíduos ficam restritos ao banco de plântulas, dependendo da abertura parcial do dossel para que seu desenvolvimento para classes de tamanho superiores seja favorecido e seu estabelecimento conduzido.

Nectandra megapotamica, Parapiptadenia rigida, Trichilia elegans, Trichilia clausseni e Sorocea bonplandii foram espécies abundantes no banco deplântulas eregeneraçãonatural estabelecida. Porém, na chuva de sementes, ocorreram em baixa densidade, no banco de sementes do solo, sequer foram encontradas. Esse fato pode estar associado à sazonalidade de produção de algumas espécies, que justifica a não observação em um único ano de estudo, além da rápida perda de viabilidade das sementes que não chegam a formar, desta maneira, banco de sementes do solo.

Assim, a perpetuação dessas espécies depende, principalmente, de condições apropriadas para germinação de sementes logo que dispersas e da presença das plantas jovens que ocorrem no subbosque da floresta.

De forma diferenciada, Phytolacca dioica e Ficus luschnathiana foram espécies arbóreas bem representadas na chuva de sementes e banco de sementes do solo, mas que não ocorreram no banco de plântulas e regeneração natural estabelecida. Apesar da ausência das espécies na vegetação jovem, estas são importantes para colonização de áreas alteradas, uma vez que possuem dormência de sementes (BACKES e IRGANG, 2002; LORENZI, 2002), podendo ficar estocadas no solo até que ocorram condições favoráveis para germinação, servindo também de atrativo para fauna.

\section{CONCLUSÕES}

O elevado número de sementes herbáceas no banco de sementes do solo, associado à elevada representatividade de espécies secundárias e clímax na chuva de sementes, banco de plântulas e regeneração natural estabelecida, permitem classificar esse remanescente no estágio de sucessão intermediário.

A chuva de sementes é um mecanismo importante para a manutenção do banco de sementes do solo e banco de plântulas, considerando a conservação das espécies arbóreas e arbustivas presentes na área de estudo e de áreas adjacentes.

O banco de sementes do solo apresenta baixa riqueza de espécies arbóreas e arbustivas, e alta riqueza e densidade de espécies herbáceas, podendo contribuir, rapidamente, na colonização inicial de áreas alteradas no remanescente.

O banco de plântulas $(\mathrm{h} \geq 30 \mathrm{~cm}$ de altura e DAP $<1 \mathrm{~cm}$ ) é composto por espécies secundárias tardias, secundárias iniciais e pioneiras, o que permite que responda a diferentes condições ambientais da floresta, representando importante mecanismo de colonização da área com espécies arbóreas e arbustivas.

A regeneração natural estabelecida $(1 \mathrm{~cm} \leq$ DAP $<5 \mathrm{~cm}$ ) é formada basicamente, por espécies secundárias tardias e pioneiras, que ocorreram em áreas com maior intensidade luminosa.

Tratos silviculturais que favoreçam a entrada de luz na floresta, como anelamento de indivíduos e eliminação de cipós, entre outros, podem proporcionar o desenvolvimento de espécies heliófilas abundantes no banco de plântulas e regeneração natural estabelecida. Já as espécies esciófilas podem ser utilizadas no enriquecimento de áreas.

As espécies Cupania vernalis, Myrocarpus frondosus, Cordia americana, Luehea divaricata, Parapiptadenia rigida, Sebastiania commersoniana e Syagrus rommanzoffiana, entre outras, podem ser espécies indicadas para a recuperação de áreas nativas na região. As espécies Gymnanthes concolor, Dasyphyllum spinescens, Trichilia elegans, Trichilia clausseni, e Sorocea bonplandii podem ser indicadas para os projetos de enriquecimento, uma vez que apresentaram abundância no sub-bosque.

\section{REFERÊNCIAS BIBLIOGRÁFICAS}

ALMEIDA, C. M.et al. Análise fitossociológica em remanescente de Floresta Estacional Decidual. Ciência Florestal, Santa Maria, 2009 (prelo).

ALMEIDA-CORTEZ, J. S. Dispersão e banco de sementes. In: FERREIRA, A. G.; BORGHETTI, F. (Org.) Germinação: do básico ao aplicado, Porto Alegre: Artmed, 2004, p. 225-235.

ARAUJO, M. M. Vegetação e mecanismos de regeneração em fragmento de Floresta Estacional Decidual Ripária, Cachoeira do Sul, RS, Brasil. 2002. 154 f. Tese (Doutorado em Engenharia Florestal)-Universidade Federal de Santa Maria, Santa Maria, 2002.

ARAUJO, M. M. et al. Caracterização da chuva de

Ci. Fl., v. 21, n. 3, jul.-set., 2011 
sementes, banco de sementes do solo e banco de plântulas em Floresta Estacional Decidual Ripária Cachoeira do Sul, RS, Brasil. Scientia Forestalis, Piracicaba, n. 66, p. 128-141, dez./2004.

BACKES, P.; IRGANG, B. Árvores do Sul: guia de identificação e reconhecimento ecológico. Porto Alegre: Pallotti, 2002. 325 p.

BAKER, H. G. Some aspects of the natural history of seed banks. In: LECK, M. A.; PARKER, V. T.; SIMPSON, R. L. (Eds) Ecology of soil seed banks. San Diego: Academic Press, 1989. p. 9-21.

BUDOWISK, G. Distribuição of tropical American rain forest species in the light of sucession process. Turrialba, San Domingos, v. 15, p. 40-42, 1965.

CARGNELUTTI FILHO, A. C. et al. Testes não paramétricos para pesquisas agrícolas. Santa Maria: UFSM/CCR/ Departamento de fitotecnia, 2001. 87 p.

CHAMI, L. B. Estudo da vegetação e mecanismos e regeneração em diferentes ambientes da Floresta Ombrófila Mista na FLONA de São Francisco de Paula, RS. 2008. 86 f. Dissertação (Mestrado em Engenharia Florestal)-Universidade Federal de Santa Maria, Santa Maria, 2008.

CROUZET, Y. Bambus. Roma: Evergreen, 1998.126 p.

FARIAS, J. A. C., et al. Estrutura fitossociológica de uma Floresta Estacional Decidual na região de Santa Maria, RS. Ciência Florestal, Santa Maria, v. 4, n. 1, p. 109-128. 1994.

FELFILI, J. M.; VENTUROLI, F. Tópicos em análise da vegetação. Brasilia: Universidade de Brasilia, 2000. 34 p.

FENNER, M; THOMPSON, K. The ecology of seeds. Cambridge: University Press, 2005. 249 p.

GARWOOD, N. C. Tropical soil seed banks: a review. In: LECK M.A.; PARKER, V. T.; SIMPSON, R. L. (Eds.) Ecology of soil seed banks. San Diego: Academic Press, 1989. p. 149-209.

LARKER, W. Ecofisiologia vegetal. São Carlos: RIMA, 2000. 531 p.

LEMOS, R. C.; AZOLIN, M. D.; ABRÃO, P. R. Levantamento de reconhecimento dos solos do estado do Rio Grande do Sul. Recife: Ministério da Agricultura, Departamento Nacional de Pesquisas Agropecuárias, Divisão de Pesquisas Pedagógicas, 1973. $431 \mathrm{p}$.

LORENZI, H. Plantas daninhas do Brasil: terrestres, aquáticas, parasitas e tóxicas. Nova Odessa: Instituto Plantarum, 1991. 140 p.

LORENZI, H. Árvores Brasileiras: Manual de identificação e Cultivo de Plantas Arbóreas Nativas do Brasil. Nova Odessa: Instituto Plantarum, 2002. 381 p. vol. 1.

MARTINEZ-RAMOS, M.; SOTO-CASTRO, A. Seed rain and advanced regeneration in a tropical rain forest. In: FLEMING; ESTRADA, A. (Eds). Frugivory and seed dispersal: Ecological and evolutionary Aspects. Dordrecht: Kluwer Academic Publishers, 1993. p. 299-318.

MELO, F. P. L. de Recrutamento e estabelecimento de Plântulas. In: FERREIRA, A. G.; BORGHETTI, F. (Org.) Germinação: do básico ao aplicado. Porto Alegre: Artmed, 2004. p 238-250.

PIZO, M., A. Padrão de deposição e sobrevivência de sementes e plântulas de duas espécies de Myrtaceae na Mata Atlântica. Revista brasileira de Botânica, São Paulo, v. 26, n. 3, p. 371-377, jul./set. 2003.

REITZ, P.; KLEIN, R. M.; REIS, A. Projeto Madeira do Rio Grande do Sul. Itajaí: Herbário Bárbara Rodrigues, 1988.525 p. Sellowia, Itajaí, n. 34-35, p. 525, 1988.

RIO GRANDE DO SUL. Governo do Estado. Inventário Florestal Contínuo do Rio Grande do Sul. Porto Alegre: FATEC/SEMA, 2002. Disponível em: (www.ufsm.br/ifcrs)> Acesso em: 17 de julho de 2008.

RIO GRANDE DO SUL. Secretária Estadual do Meio Ambiente. Departamento de Florestas e Áreas Protegidas. Diretrizes ambientais para restauração de matas ciliares. Porto Alegre: SEMA, 2007. 33 p.

ROIZMAN, L. G. Fitossociologia e dinâmica do banco de sementes do solo de populações arbóreas de floresta secundária em São Paulo, SP. 1993. 156 f. Dissertação (Mestrado em Ecologia) Universidade de São Paulo, São Paulo, 1993.

RUDGE, A. de C. Contribuição da chuva de sementes na recuperação de áreas e do uso de poleiros como técnica catalisadora da sucessão natural. 2008. 113 f. Dissertação (Mestrado em Ciências Ambientais e Florestais)-Universidade Federal Rural do Rio de Janeiro, Rio de Janeiro, 2008.

SILVA, W. C., et al. Estudo da regeneração natural de espécies arbóreas em fragmento de Floresta Ombrófila Densa, Mata das Galinhas, no município de Catende, Zona da Mata Sul de Pernambuco. Ciência florestal, Santa Maria, v.17, n. 4, p. 321331, out./dez. 2007.

SIMPSON, R. L. et al. Seed banks: general concepts and a methodological issues. In: LECK, M. A.; PARKER, T. V.; SIMPSON, R. L. (Eds) Ecology of 
soil seed banks. New York: Academic Press, 1989, VIEIRA, I. C. G. Florest succession after shifting p. 3-8. cultivation in eastern Amazonia. 1996. 205

STRECK, E. D. et al. Solos do Rio Grande do Sul. f. Thesis (Doctor of Philosophy)-University of Porto Alegre: EMATER/RS-ASCAR, 2008. 222 p. Stirling, Scotland, 1996. 